\title{
New Microbiological and Pharmacokinetic models
}

Jose I. Bueso-Bordils*, Pedro A. Alemán-López, Sara Costa-Piles, Maria J. Duart, Luis Lahuerta-Zamora, Rafael Martín-Algarra and Gerardo M. Antón-Fos.

Department of Pharmacy, Faculty of Health Sciences, CEU Cardenal Herrera University, P.O. Box:46113, Moncada, Spain

Abstract. Abstract: In this paper, a multilinear regression (MLR) analysis has been carried out in order to accurately predict physicochemical properties and biological activities on a group of antibacterial quinolones by means of a set of structural descriptors called topological indices. The aim of this work is to develop prediction equations for these properties after collecting the maximum number of data from the literature on antibacterial quinolones. The five regression functions selected by presenting the best combination of various statistical parameters, subsequently validated by means of internal validation (intercorrelation, Yrandomization and leave-one-out cross-validation tests), allowed the reliable prediction of minimum inhibitory concentration 50 versus Staphylococcus aureus (MIC50Sa), Streptococcus pyogenes (MIC50Spy) and Bacteroides fragilis (MIC50Bf), mean residence time (MRT) after oral administration and volume of distribution $\left(\mathrm{V}_{\mathrm{D}}\right)$. We conclude that the combination of molecular topology methods and MLR provides an excellent tool for the prediction of pharmacological properties.

\section{Graphical Abstract}

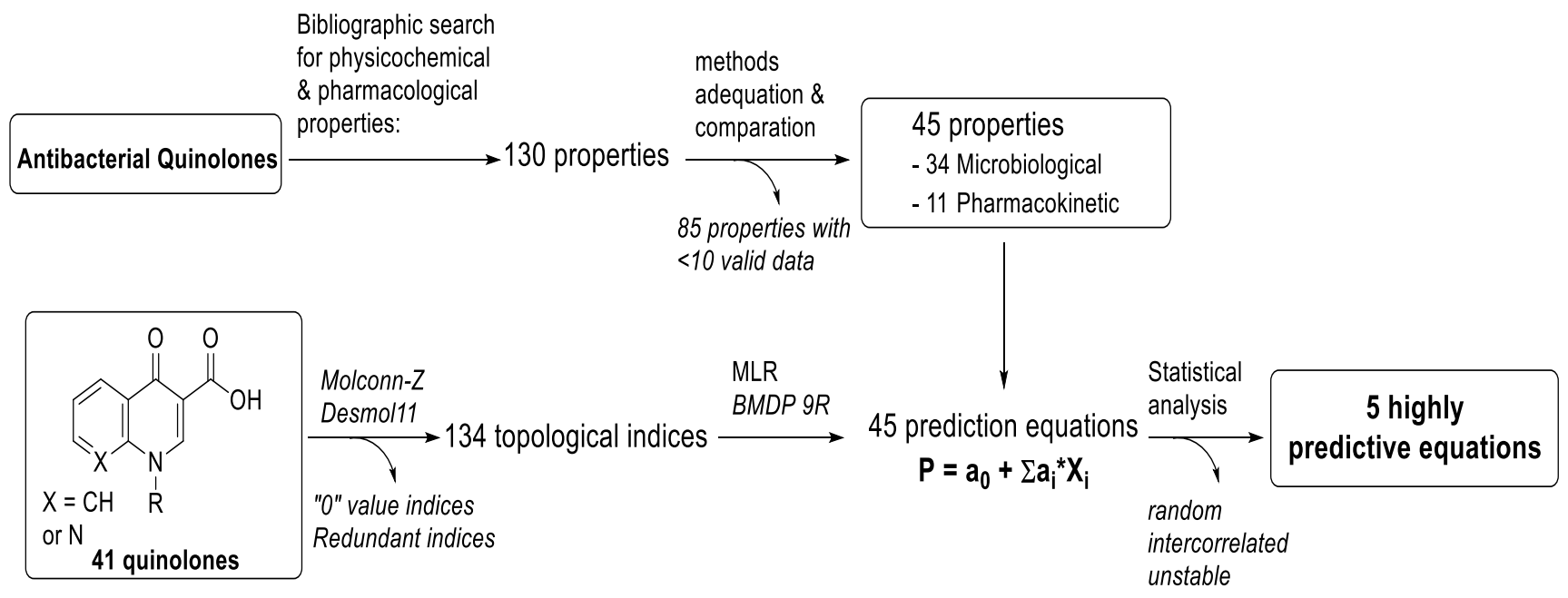

Keywords: molecular topology, multilinear regression (MLR), molecular connectivity, topological indices, quinolones, QSAR.

\section{References}

[1] Khardori, N. Antibiotics-past, present, and future. Med. Clin. North Am., 2006, 90(6), 1049-1076.

[2] Spellberg, B.; Bartlett, J.G.; Gilbert, D.N. The future of antibiotics and resistance. N. Engl. J. Med., 2013, 368(4), 299-302.

[3] Alvan, G.; Edlund, C.; Heddini, A. The global need for effective antibiotics-a summary of plenary presentations. Drug Resist. Update, 2011, 14(2), 70-76.

[4] Drlica, K.; Malik, M.; Kerns, R.J.; Zhao, X. Quinolone-mediated bacterial death. Antimicrob. Agents Chemother., 2008, 52(2), 385-392.

[5] Mitscher, L.A. Bacterial topoisomerase inhibitors: quinolone and pyridone antibacterial agents. Chem. Rev., 2005, 105(2), 559-592.

[6] Andersson, M.I.; MacGowan, A.P. The quinolones: decades of development and use. J. Antimicrob. Chemother., 2003, 51(S1), 1-11. 
[7] Cherkasov, A.; Muratov. E.N.; Fourches, D.; Varnek, A.; Baskin, I.I.; Cronin, M.; Dearden, J.; Gramatica, P.; Martin, Y.C.; Todeschini, R.; Consonni, V.; Kuz'min, V.E.; Cramer, R.; Benigni, R.; Yang, C.; Rathman, J.; Terfloth, L.; Gasteiger, J.; Richard, A.; Tropsha, A. QSAR modeling: where have you been? Where are you going to? J. Med. Chem., 2014, 57(12), 4977-5010.

[8] Karelson, M. Molecular Descriptors in QSAR/QSPR, John Wiley \& Sons, New York, 2000.

[9] Todeschini, R.; Consonni, V. Molecular Descriptors for Chemoinformatics, vol. 41; Wiley VCH Verlag GmbH: Weinheim, 2009.

[10] Rabal, O.; Borrel, J.I.; Teixidó, J. Cribado virtual: aproximaciones y técnicas. Afinidad, 2007, 64(529), 329-345.

[11] Pastor, M.; Álvarez-Builla, J. Técnicas QSAR en diseño de fármacos. An. Real Acad. Farm., 1994; I, 69-98.

[12] Tropsha, A. Best practices for QSAR model development, validation, and exploitation. Mol. Inform., 2010, 29(6-7): 476-488.

[13] Hawkins, D.M. The problem of overfitting. J. Chem. Inf. Comput. Sci., 2004, 44(1), 1-12.

[14] Hansch, C.; Maloney, P.P.; Fujita, T.; Muir, R.M. Correlation of biological activity of phenoxyacetic acids with Hammett substituent constants and partition coefficients. Nature, 1962, 194(4824), 178-180.

[15] Hansch, C.; Leo, A. Substituent Constants for Corrleation Analysis in Chemistry and Biology, Wiley: New York, 1979.

[16] Free, S.M.; Wilson, J.W. A mathematical contribution to structure-activity studies. J. Med. Chem., 1964, 7(4): 395-399.

[17] Lipinski, C.A. Lead- and drug-like compounds: the rule-of-five revolution. Drug Discov. Today. Technol., 2004, 1(4), 337-341.

[18] Huggins, D.J.; Venkitaraman, A.R.; Spring, D.R. Rational methods for the selection of diverse screening compounds. ACS Chem. Biol., 2011, $6(3), 208-217$.

[19] Roy, K.; Kar, S.; Das, R.N. A Primer on QSAR/QSPR Modeling: Fundamental concepts, $1^{\text {st }}$ ed.; Springer, 2015.

[20] Singh, N.; Sun, H.; Chaudhury, S.; Abdulhameed, M.D.; Wallqvist, A.; Tawa, G. A physicochemical descriptor-based scoring scheme for effective and rapid filtering of kinase-like chemical space. J. Cheminform., 2012, 4(1), 4.

[21] Kier, L.B.; Hall, L.H. Molecular Connectivity in Chemistry and Drug Research, Academic Press: New York, 1976.

[22] Ovidiu, I. Chemical graphs, molecular matrices and topological indices in chemoinformatics and quantitative structure-activity relationships. Curr. Comput.-Aided Drug Des., 2013, 9(2), 153-163.

[23] Hert, J.; Willett, P.; Wilton, D.J.; Acklin, P.; Azzaoui, K.; Jacoby, E.; Schuffenhauer, A. New methods for ligand-based virtual screening: use of data fusion and machine learning to enhance the effectiveness of similarity searching. J. Chem. Inf. Model., 2006, 46(2), 462-470.

[24] Yu, N.; Bakken, G.A. Efficient exploration of large combinatorial chemistry spaces by monomer-based similarity searching. J. Chem. Inf. Model., 2009, 49(4), 745-755.

[25] Yang, G.F.; Huang, X. Development of quantitative structure-activity relationships and its application in rational drug design. Curr. Pharm. Des., 2006, 12(35), 4601-4611.

[26] Gálvez J, de Julián-Ortiz JV, García-Domenech R. General topological patterns of known drugs. J. Mol. Graphics, 2001; 20(1), 84-94.

[27] López-Malo, D.; Bueso-Bordils, J.I.; Duart, M.J.; Alemán-López, P.A.; Martín-Algarra, R.V.; Antón-Fos, G.M.; Lahuerta-Zamora, L.; MartínezCalatayud, J. QSPR studies on the photoinduced-fluorescence behavior of pharmaceuticals and pesticides. SAR QSAR Environ. Res., 2017, 28(7), 609-620.

[28] Zanni, R.; Galvez-Llompart, M; Morell, C.; Rodríguez-Henche, N.; Díaz-Laviada, I.; Recio-Iglesias, M.C.; Garcia-Domenech, R.; Galvez, J. Novel cancer chemotherapy hits by molecular topology: dual Akt and Beta-catenin inhibitors. PLoS One, 2015, $10(4)$, e0124244.

[29] Afantitis, A.; Melagraki, G.; Sarimveis, H.; Koutentis, P.A.; Markopoulous, J.; Igglessi-Mrkopoulou, O. A novel simple QSAR model for the prediction of anti-HIV activity using multiple regression analysis. Mol. Divers., 2006, 10(3), 405-414.

[30] Bueso-Bordils, J.I.; Alemán, P.A.; Zamora, L.L.; Martín-Algarra, R.; Duart, M.J.; Antón-Fos, G.M. Topological model for the search of new antibacterial drugs. 158 theoretical candidates. Curr. Comput. Aided Drug Des., 2015, 11(4), 336-345.

[31] Bueso-Bordils, J.I.; Perez-Gracia, M.T.; Suay-Garcia, B.; Duart, M.J.; Martin Algarra, R.V.; Lahuerta Zamora, L; Anton-Fos, G.M.; Aleman Lopez, P.A. Topological pattern for the search of new active drugs against methicillin resistant Staphylococcus aureus. Eur. J. Med. Chem., 2017, $138,807-815$.

[32] CLSI. Performance standards for antimicrobial susceptibility testing; twenty-second informational supplement. CLSI document M100-S22; 32(3). Wayne, PA: Clinical and Laboratory Standards Institute; 2012.

[33] DESMOL11 software; Unidad de Investigación de Diseño de Fármacos y Conectividad Molecular, Facultad de Farmacia, Universitat de València: Valencia (Spain).

[34] Hall, L.H. MOLCONN-Z software; Eastern Nazarene College: Quincy (Massachusetts), 1995.

[35] Dixon, W.J. BMDP Statistical software; University of California: Berkeley, 1990.

[36] Furnival, G.M.; Wilson, R.W. Regressions by leaps and bounds. Technometrics 1974, 16(4), 499-511.

[37] Hocking, R. Criteria for selection of a subset regression: which one should be used? Technometrics 1972, 14(4), 967-970. 